\section{D Seismic Reflection and VSP for Hydrogeology} Brett Harris*, Milovan Urosevic, Anton Kepic

\section{Summary}

Modern hydrogeology is increasingly depending numerical modeling to simulate impacts of water resource projects tens or hundreds of years into the future. The validity of such numerical models is highly dependent on accurate distribution of key hydrogeological parameters. A combination of 3D seismic reflection and Vertical Seismic Profiling (VSP) offers the potential to create detailed, accurate, hydrostratigraphic frameworks within which physical, chemical and biological properties can be distributed. We provide the outline and outcomes from a systematic research program designed to develop and optimize 3D seismic reflection and VSP techniques tailored for hydrogeology. The test site is located at the Beenyup Waste Water Treatment Plant near a major freeway, where a long term high volume purified waste water injection trial will commence in 2009.

\section{Introduction}

Water management is a sophisticated scientific discipline. Organizations that may influence water balance by their activities are often required to make detailed future predictions based on numerical modeling of hydraulic flow, solute transport and/or reactive transport. The validity of such predictions is highly dependent of the correct distribution of physical, chemical, and/or biological properties throughout millions of cubic meters of earth. Seismic reflection offers the potential to recover the detailed framework within which hydrological parameter can be correctly distributed. Areas in which 3D seismic reflection and VSP should be of tremendous value include; Aquifer Storage and Recovery, Managed Aquifer Recharge, well field development, and mine dewatering.

\section{Method}

A 3D seismic survey has been completed and processed at the Beenyup Test Site and a detailed image for a 300 by 300 by $300 \mathrm{~m}$ cube has been generated. The target zone was from surface to $\sim 300 \mathrm{~m}$ below ground level. CDP bin size for the depth converted image is $2 \mathrm{~m}$ by $2 \mathrm{~m}$ by $2 \mathrm{~m}$. The seismic data provides at least seven distinct interfaces that are correlated with geophysical logs and core. Of particular importance is the clear continuity of the upper and lower seals that confine the primary injection zone (i.e. the aquifer into which purified waste water will be injected). This is a key practical outcome for the 3D seismic as it adds weight to the proposition that the injected water will move radial out and so be entirely contained in the target aquifer.
The VSP research program is systematical analysing a large number of combinations of source/receiver type and survey parameters to establish effective and practical methods for Hydrogeology. The potential benefits that VSP offers hydrogeology include:

(i) Recovery of detailed near well stratigraphy.

(ii) Recovery true dip information (i.e. from 3D-VSP) on hydrostratigraphic units. Dip information can be a key input to modern stratigraphic modeling packages.

(iii) Recovery of subsurface images from multiple walk away VSP surveys. Note that VSP can be completed in multiple monitoring bores, located around production wells

(iv) Depth conversion for 3D and 2D seismic reflection surveys.

(v) Full wave form recovery. This may be important for design of $\mathrm{Q}$ compensation and/or attribute design for $3 \mathrm{D}$ and $2 \mathrm{D}$ seismic data sets.

Research topics that are currently being addressed include:

(i) An examination of the relative merits of hydrophones versus $3 \mathrm{C}$ Geophones.

(ii) Identification of most suitable source type (e.g. Mini Vibrator versus Drop Hammer)

(iii) Removal and/or use of passive seismic "noise"

(iv) Shear wave versus $P$ wave sources.

(v) Design of "hydrogeological" attributes from VSP and application to 3D seismic imaging.

(vi) A comparison of VSP data from a range of cased wells and uncased drill holes

(vii) The application of 4D VSP for assessing velocity versus pressure during the injection test

\section{Examples (3D Seismic and VSP)}

Figure 1, shows a small sub-cube and two surfaces obtained from the 3D seismic volume at Beenyup. The two surfaces clearly delineated the top and bottom of the aquifer unit (i.e. injection zone). These surfaces were obtained by selecting and tracking the character of a single wiggle trace across the data set. In summary the injection zone (aquifer) and upper and lower confining layer were exceedingly well characterized by the 3D seismic data. 


\section{D Seismic and VSP for Hydrogeology}

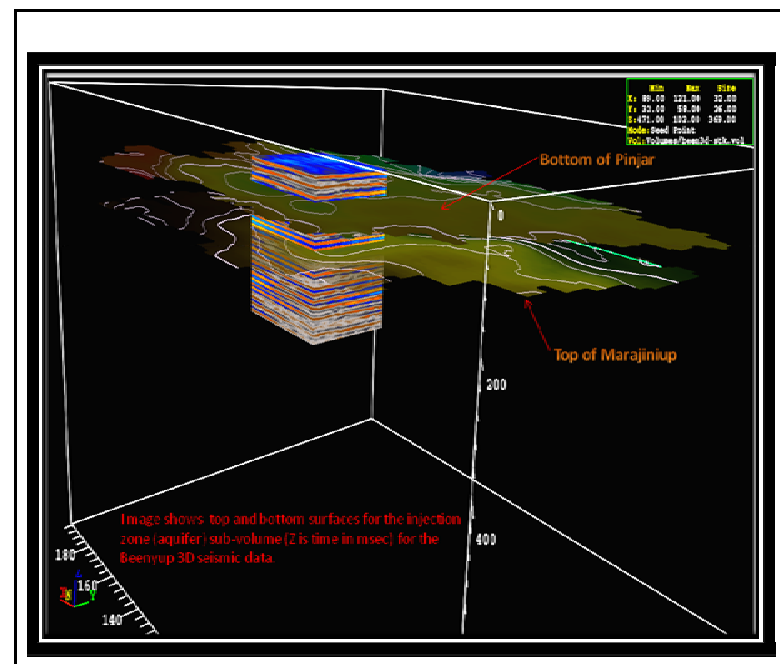

Figure 1: Image of small cube sliced out the Beenyup 3D seismic volume and upper and lower surfaces for the injection zone. The entire upper and lower surfaces were automatically picked based on a single pilot trace. The above image is obtained using the Landmark GeoProbe Software.

An example of zero offset VSP shot record from a hydrophone string, lowered into a large diameter water well, is provided in Figure 2.

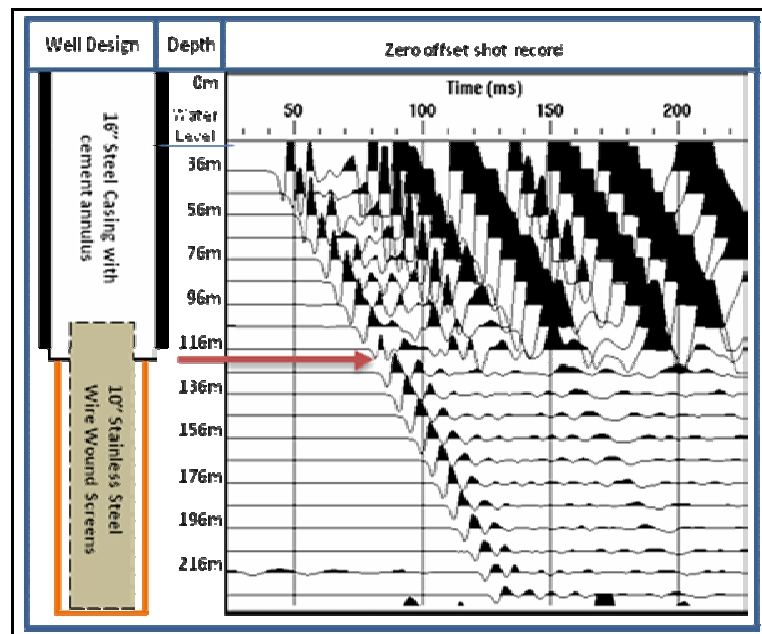

Figure 2: Shot Record for zero offset VSP obtained from a hydrophone string lower into a large diameter water well. The traces are unscaled and horizontal. They are shown against well design and depth. There is a clear partician between 16 inch blank steel casing and wire wound stainless steel screens at about $126 \mathrm{~m}$. Zero offset VSP with a hydrophone string in a large diameter water well represents a highly changing situation.
The hydrophones located in the 16" blank steel casing are clearly dominated by tube waves. However the VSP is dramatically improved for the hydrophones located within the wire wound stainless steel screens that have open area and hence direct communication with the formation. The first breaks remain well resolved over all the hydrophones.

Many other VSP experiments have been completed with both the cemented 3C geophone string and hydrophone string. These are progressive demonstrating the value and limitations, and application of VSP in Hydrogeology.

\section{Conclusions}

A comprehensive research program targeted at developing efficient 3D seismic and VSP for hydrogeology has been developed. The project is delivering experiments and examples demonstrating the importance and practicality of seismic reflection in hydrogeology

The 3D seismic reflection data from the Beenyup high volume purified waste water injection site has provided a quality 3D representation of all key sand and clay dominated layers (i.e. aquifers and aquacludes). The 3D data was acquired and processed at a small fraction of the total cost of drilling at the Beenyup site.

Many comparisons of VSP data have been obtained from both a $3 \mathrm{C}$ cemented geophone string and a Hydrophone String lowered into water wells of difference design and dimensions at the Beenyup test site. The hydrophone string has proven to be rapid and easy to deploy and provides accurate velocity information (i.e. first breaks). The 3C geophone string and under certain circumstances the hydrophone string also provide a clear image of sand and clay distribution in the near well environment. This is of particular importance for injection projects. The research program will continue until at least 2011.

\section{Acknowledgments}

We would like to thank the Water Corporation of Western Australia for supporting this important research in hydrogeophysics. We would also thank Curtin Universitie's Centre for High Definition Geophysics for providing infrastruacture and equipment to complete the many surveys and experiments. 